\title{
Dynamic Work Planning by Using Simulation-based Optimization in Consideration of Workers' Skill and Training*
}

\author{
Takeshi TATEYAMA**, Toshitake TATENO*** and Keiko SHIMIZU ${ }^{\dagger}$ \\ ** Tokyo Metropolitan University \\ 6-6 Asahigaoka, Hino-shi, Tokyo 191-0065, Japan \\ E-mail: tateyama@sd.tmu.ac.jp \\ *** Advanced Institute of Industrial Technology \\ 1-10-40 Higashioi, Shinagawa-ku, Tokyo 140-0011, Japan \\ $\dagger$ East Japan Railway Company \\ 2-2 Yoyogi, Shibuya-ku, Tokyo 151-0053, Japan
}

\begin{abstract}
High-skill tasks such as aircraft maintenance procedures generally require considerable on-the-job training (OJT). Because OJT requires a considerable amount of time, longterm scheduling of OJT by considering both working efficiency and skill development is required. The authors have proposed education coefficient (EC) as a parameter that adjusts the frequency of OJT and have proposed a scheduling support system for longterm scheduling of OJT considering working efficiency and skill education. Users of the proposed scheduling support system can easily adjust the balance between working efficiency and skill education by changing EC. However, it is difficult for them to find effective values of EC that realize a good balance between working efficiency and skill education to maintain a certain number of skilled workers. In this paper, we propose a simulation-based scheduling optimization system using a simple genetic algorithm (GA). The purpose of this study is to adjust the value of EC automatically by considering the balance between working efficiency and skill education and to find effective rules for adjusting the value of EC. The experimental results show that the proposed system computes solutions that are better than those computed by using a constant EC.
\end{abstract}

Key words : Scheduling, On the Job Training (OJT), Dynamic Work Planning, Simulation, Genetic Algorithm (GA)

\section{Introduction}

For the maintenance of heavy machinery (e.g., aircraft jet engines), the complex nature of the tasks and the high level of skill required necessitates on-the-job training (OJT). Because OJT requires a considerable amount of time, long-term scheduling of OJT by considering both working efficiency and skill development is required. Generally, operational managers have to allocate such tasks to workers by using a trial-and-error method. However, this is becoming very difficult because of the increasing complexity of products and the mobility of human resources. To overcome this problem, we have proposed a scheduling support system for long-term scheduling of OJT by considering working efficiency and skill education ${ }^{(1)}$. This support system includes worker models based on the LFCM model ${ }^{(2)}$ to simulate learning and forgetting, an agent-based scheduling algorithm, and a simulation system. By using this support system, we can simulate and evaluate dynamic scheduling for the maintenance of heavy machinery. In addition, we have proposed education coefficient (EC) as a parameter that adjusts the frequency of OJT. Users of the proposed scheduling support system can easily adjust the balance between working efficiency and skill education by changing EC; further,

*Received 31 0ct., 2009 (No. 09-0655) [DOI: 10.1299/jamdsm.4.597]

Copyright (c) 2010 by JSME 
they can evaluate the results of scheduling by executing the simulation. However, it is difficult for them to find effective values of EC that realize a good balance between working efficiency and skill education to maintain a certain number of skilled workers. In this paper, we propose a simulation-based scheduling optimization system using a simple genetic algorithm $(\mathrm{GA})^{(3)}$. The purpose of this study is to adjust the value of EC automatically by considering the balance between working efficiency and skill education and to find effective rules for adjusting the value of EC. The experimental results show that the proposed system computes solutions that are better than those computed by using a constant EC.

\section{Problem Definition}

In this study, we deal with the scheduling of the maintenance of heavy machinery. We assume $N_{w}$ workers do maintenance on $N_{m}$ machines within $L$ [years]. The maintenance for a machine $m_{i}\left(i=1,2, \ldots, N_{m}\right)$ includes $N_{j}$ jobs in $J_{\text {all }}$ jobs $\left(J_{\text {all }}\right.$ is the total number of types of jobs). Each job $j_{i}\left(i=1,2, \ldots, N_{j}\right)$ requires $t_{j}[\mathrm{~h}]$ to be completed. The workers perform these jobs in parallel. Each worker $W_{i}$ has job level $l v_{j}^{i}\left(l v_{j}^{i}=1\right.$ or 2$)$ for each job $j$. A worker Wi whose job level $l v_{j}^{i}$ is 2 can perform the job $j$ by himself. On the other hand, when his job level $l v_{j}^{i}$ is 1 , he requires OJT to perform and learn the job $j$. In OJT, he performs the job $j$ with one supervisor whose job level for the job $j$ is 2. OJT for job $j$ requires $H_{O J T}\left(H_{O J T}>1\right) \times t_{j}$ [h] to complete. Because OJT requires more time than if the task were performed by level 2 workers, there is a trade-off between reduction of hours of work and skill education by OJT. The workers' job levels increase from 1 to 2 by performing OJT $N_{u}$ times within $T_{u}$ years. In contrast, the job level goes back from 2 to 1 when the workers have not performed the job for $T_{d}$ years. They need to perform the job periodically in order to maintain their job level.

\section{Dynamic Work Planning}

\subsection{Agent-Based Dynamic Scheduling Method}

In this study, the purpose of scheduling is to allocate jobs to workers dynamically in consideration of working efficiency, skill education, and changes in the situation from a longterm perspective. We use an agent-based dynamic scheduling method using the "contact net protocol"(4) to do so. The following is the procedure of the job allocation algorithm:

Step 1: A "manager agent" requests job information from every "job agent."

Step 2: The job agents respond with the job information.

Step 3: The manager agent lists the possible jobs in the order of ascending priority.

Step 4: The manager agent announces the job information to all "worker agents".

Step 5: Each worker agent calculates "estimated completion time (ECT)" using the following equations:

$$
\begin{aligned}
& E C T=E C T_{c}+P T-E C[h] \\
& E C= \begin{cases}E C_{c} & \text { if Level }\left(j_{j}, W_{k}\right)=1 \\
0 & \text { else }\end{cases}
\end{aligned}
$$

Here, $E C T_{c}$ is the estimated completion time of contracted jobs, and $P T$ is the processing time for the announced job for each worker agent. $\operatorname{Level}\left(j_{j}, W_{k}\right)$ is a function with arguments such as job ID $j_{j}$ and a worker agent's ID $W_{k}$; this function computes the job level. $E C$ is the "education coefficient" that we proposed in the previous work. $E C$ is a parameter that is used for controlling the frequency of OJT in the scheduling. The value of $E C$ is a constant value $E C_{c}$ tuned by the user (however, in the new method proposed in this paper, the value of EC automatically changes depending on the situation) when the job level of the worker agent is 2; the value of $E C$ is zero when the job level is 1 . When the value of $E C$ is high, the frequency of OJT increases. In contrast, when the value of $E C$ is low, the frequency decreases. $E C$ is described in detail in the next section. After computing $E C T_{c}$, the worker agents send the $E C T_{c}$ to the manager agent. 


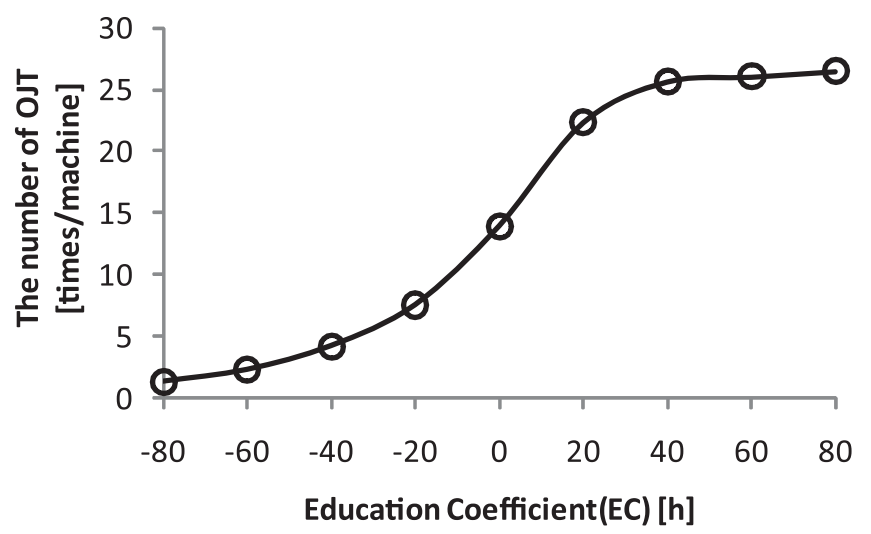

Fig. 1 Changes in the number of OJTs [times/machine] with changes in the value of EC

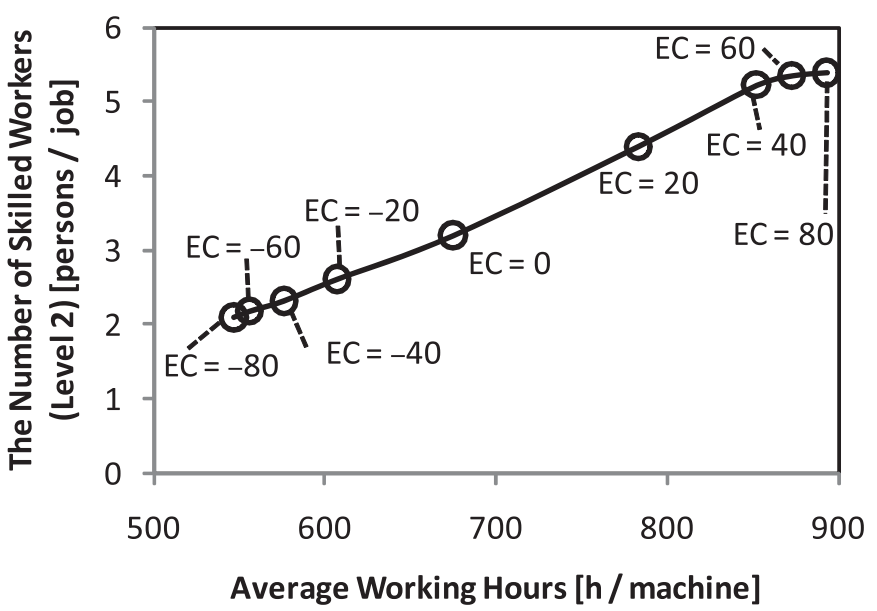

Fig. 2 Sets of average working hours and the number of skilled workers computed by using different values of EC

Step 6: The manager agent contracts the job to the worker agent whose $E C T_{c}$ value is the lowest and requests the other agents to cancel the job.

Step7: The manager agent enters the job information into the scheduling chart and requests the job agent to change the work record.

The process from Step 1 to Step 7 is repeated until all jobs are allocated.

\subsection{Characteristic Features of EC}

This section describes EC in relation to the results of scheduling. As described in the foregoing section, EC is a parameter that is used for controlling the frequency of OJT in scheduling. We treated EC as a constant parameter in the earlier study. Figure 1 shows the relation between values of EC and the number of OJTs (refer to $\$ 5$ for details of this experiment). This figure indicates that the number of OJTs increases with an increase in the value of EC. Figure 2 shows the relation between values of EC and the average working hours [h/machine] and the average number of workers whose job level is 2 [persons/job], respectively. These figures indicate the trade-off between the reduction of working hours and the increase in the number of skilled workers. Therefore, we need to adjust the value of EC by considering a balance between them. 


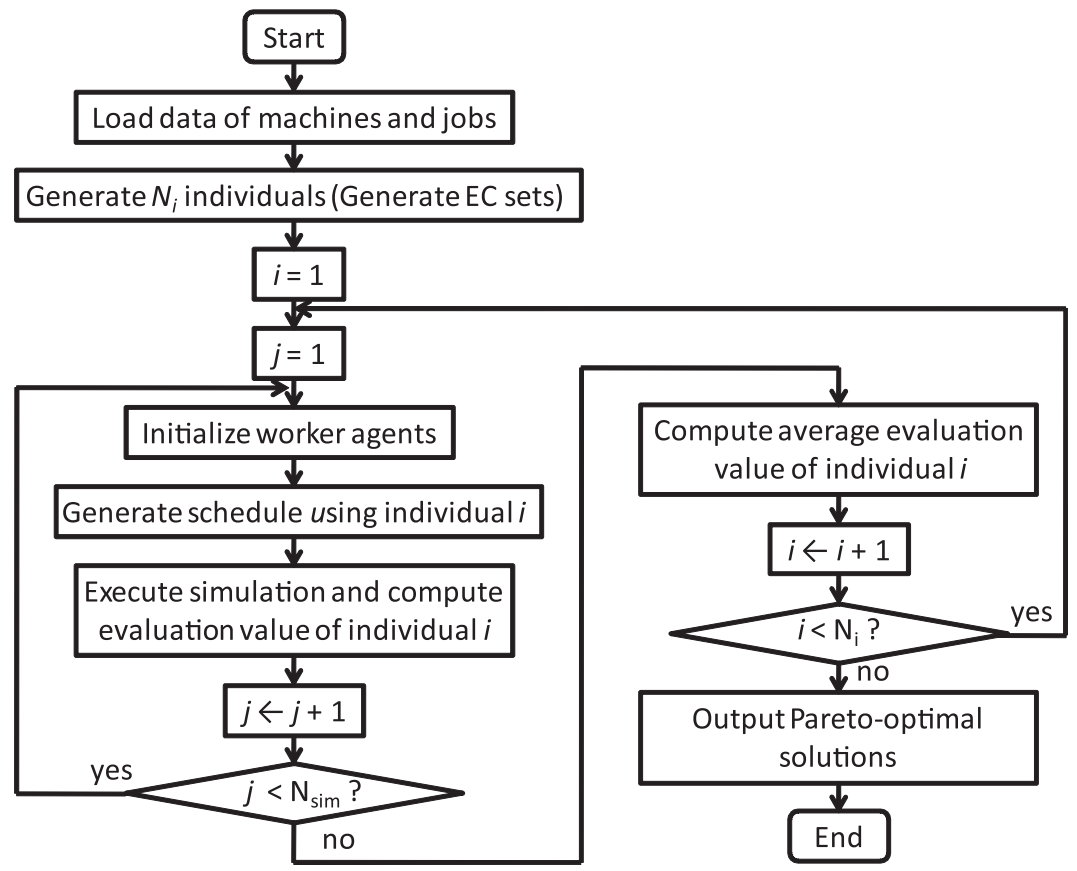

Fig. 3 Simulation-based optimization system using a genetic algorithm

\section{Simulation-Based Optimization by Using Genetic Algorithm}

The purpose of this study is to adjust the value of EC automatically by considering the balance between working efficiency and skill education and to find effective rules for adjusting the value of EC. In this paper, we propose a simulation-based optimization system by using a genetic algorithm (GA). GA is often applied to scheduling problems. For example, Tamaoki et al. have applied GA to a production scheduling problem in order to find effective rules for generating good scheduling ${ }^{(5)}$. In this system, the value of EC changes each time the manager agent allocates a certain number of jobs to the worker agents. This method will enable us to get more effective scheduling results than if the value of EC were constant. Figure 3 indicates the outline of the proposed system. In this figure, $N_{i}$ is the number of individuals. $N_{\text {sim }}$ is the number of executions of simulation required to evaluate an individual. $i$ is the ID of an individual, and $j$ is the sample number of the simulation. This system searches effective sets of values of EC on the basis of a genetic algorithm and by repeated simulations. The following sections describe the details of the proposed system.

\subsection{GA Coding}

An individual whose chromosome length is $L_{c}$ has $L_{c}$ information about values of EC. In this system, for the sake of simplicity, the value of EC changes every time $N_{m} N_{j} / L_{c}$ jobs are allocated. The values of the individuals are discrete values ranging from $E C_{\min }$ to $E C_{\max }$. $N_{E C}$ denotes the total number of discrete EC values. The initial values of EC are randomly set when the individuals are generated. Figure 4 shows the outline of the construction of the individuals in the system. In the example shown in Fig. $4, E C_{\min }$ is $-40, E C_{\max }$ is 60 , and $N_{E C}$ is 6 (the set of EC values: $-40,-20,0,20,40,60$ ).

\subsection{Fitness of the Individuals}

The purpose of this system is to get effective EC sets that help to decrease working hours and maintain the number of skilled workers above a certain number in order to respond to the skilled workers' sudden illnesses or retirement. Therefore, we apply two values computed by 


\section{Chromosome Length $L_{c}$}

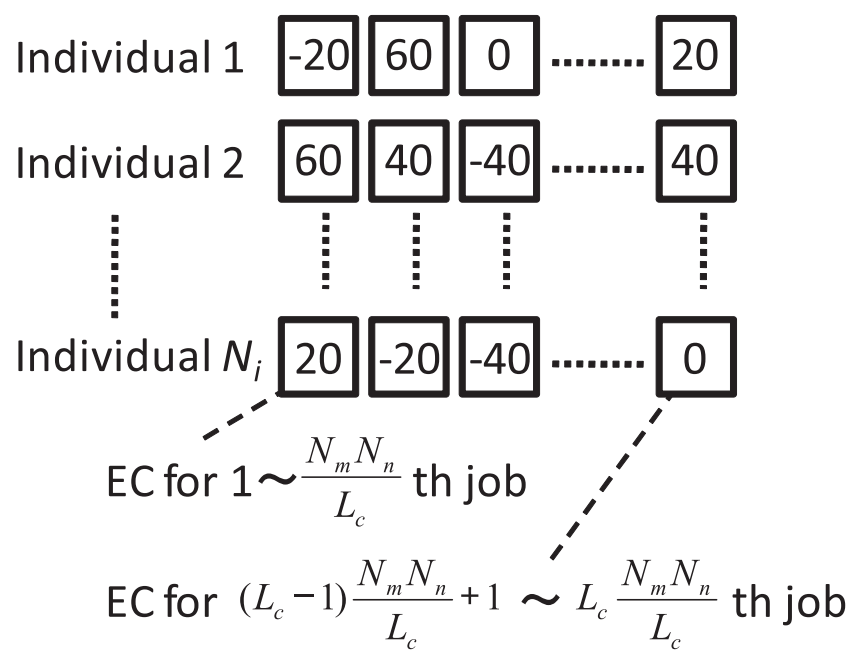

Fig. 4 GA Coding in the proposed system

using the following equations as the fitness of the individuals:

$$
\begin{aligned}
& F_{1}^{i}=\frac{365 \times 24 \times L}{T_{\text {all }}^{i}} \\
& F_{2}^{i}=1-\frac{1}{J_{\text {all }}} \sum_{d=1}^{D_{\text {all }}} \frac{N_{J E L}(d)}{D_{\text {all }}}
\end{aligned}
$$

Here, $T_{\text {all }}^{i}$ corresponds to the working hours required to perform all jobs of all machines by using a set of ECs written in individual $i, N_{J E L}(d)$ is (in this paper, the threshold value is taken to be 2) on the $d_{t h}$ day from the start date of the scheduling. Therefore, the problem to be solved is a multi-objective optimization problem. In each crossover operation of GA, two individuals are selected as parents by using a roulette selection method on the basis of the following evaluated values:

$$
E_{i}=w_{1} F_{1}^{i}+w_{2} F_{2}^{i}
$$

Here, $E_{i}$ is the evaluated value of individual $i$, and $w_{1}$ and $w_{2}$ correspond to the weights of fitness. These values are determined by the users on the basis of the degrees of importance.

\subsection{Crossover and Mutation}

The proposed system uses a simple single-point crossover method. The crossover point is randomly determined every time the system executes a crossover. The mutation algorithm is executed as follows: In each crossover operation, an EC value in a gene of an individual changes to another value with probability $R_{m}$. This system treats finite and discrete EC values, and the new EC value is randomly selected from among $N_{E C}$ values in the EC value set.

\subsection{Scheduling and Simulation}

The system executes scheduling by using a set of ECs written in each individual, and it runs the simulation several times in order to compute the fitness of each individual. The simulation is executed several times using different initial settings (initial job levels of each worker agent, etc.), and the average evaluated value of each individual is computed. 


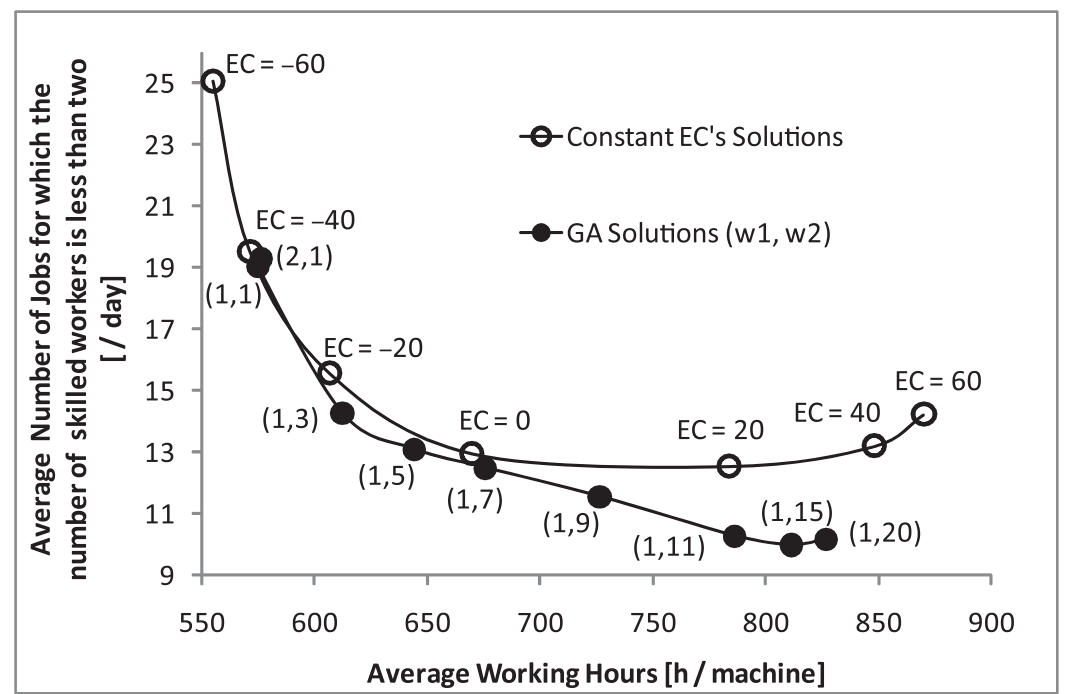

Fig. 5 Pareto-optimal solutions calculated by the proposed system and solutions calculated by using a constant EC

\subsection{Pareto-Optimal Solutions}

After several GA operations, the proposed system computes a set of preferred solutions and outputs some Pareto-optimal solutions. The users select one individual to apply based on the solutions.

\section{Experiments and Results}

This section describes the effectiveness of the proposed system on the basis of some experiments. The experiments are performed by using the following parameters: The number of worker agents $N_{w}=8$, the number of machines $N_{m}=100$, the number of jobs included in one machine's maintenance $N_{j}=100$, and scheduling period $L=10$ [years]. The number of working hours for each job $j t_{j}[\mathrm{~h}]$ is determined by using a random number $\left(1 \leq t_{j} \leq 120\right)$. The number of working hours for OJT is $H_{O J T} \times t_{j}=1.5 \times t_{j}$. Initial job levels of each worker agent are determined using random numbers as follows: $25 \%$ of 100 jobs $\leftarrow$ level $2,75 \%$ of 100 jobs $\leftarrow$ level 1 . The worker agents can rise to job level 2 from level 1 by performing OJT $N_{u}=4$ [times] within $T_{u}=4$ [years]. Their job level falls from 2 to 1 when they have not performed the job for $T_{d}=4$ [years]. The parameters for GA are set as follows: chromosome length $L_{c}=10$, the number of individuals $N_{i}=100$, and mutation rate $R_{m}=0.03$. The authors have tuned the above parameters for GA in the preliminary experiments. The value of EC written in each gene is a discrete value $(-60,-40,-20,0,20,40,60)$. The simultor outputs a different result each time the simulation is excuted because the initial job levels of each worker agent are reset at the beginning of each simulation. Therefore, we perform the simulation 10 times (ten years simulation $\times 10$ times) in order to calculate the fitness of each individual and evaluate it. We evaluate the solutions obtained by the proposed method by comparison with the solutions calculated by the simulation using constant $\mathrm{EC}$ values. For comparison, we apply following seven constant $\mathrm{EC}$ values: $\mathrm{EC}=(-60,-40,-20,0,20,40,60)$. Figure 5 shows nine sets of average Pareto-optimal solutions obtained by performing GA ten times and seven sets of average solutions calculated by using above seven constant EC values. The horizontal axis indicates the average working hours per machine $x[\mathrm{~h} /$ machine] and the vertical axis indicates the number of jobs for which the number of skilled workers is less than two $y$ [jobs]. The GA solutions $(x, y)$ are calculated when the sets of weights of evaluated values described in the $\S 4.2$ are equal to $\left(w_{1}, w_{2}\right)=(2,1),(1,1),(1,3),(1,5),(1,7),(1,9),(1,11),(1,15),(1,20)$. By executing GA once, one individual whose fitness is the highest in the population is selected. A set of average solution $\left(\overline{x_{k}}, \overline{y_{k}}\right)$ of the selected individual $k$ is calculated by performing the 


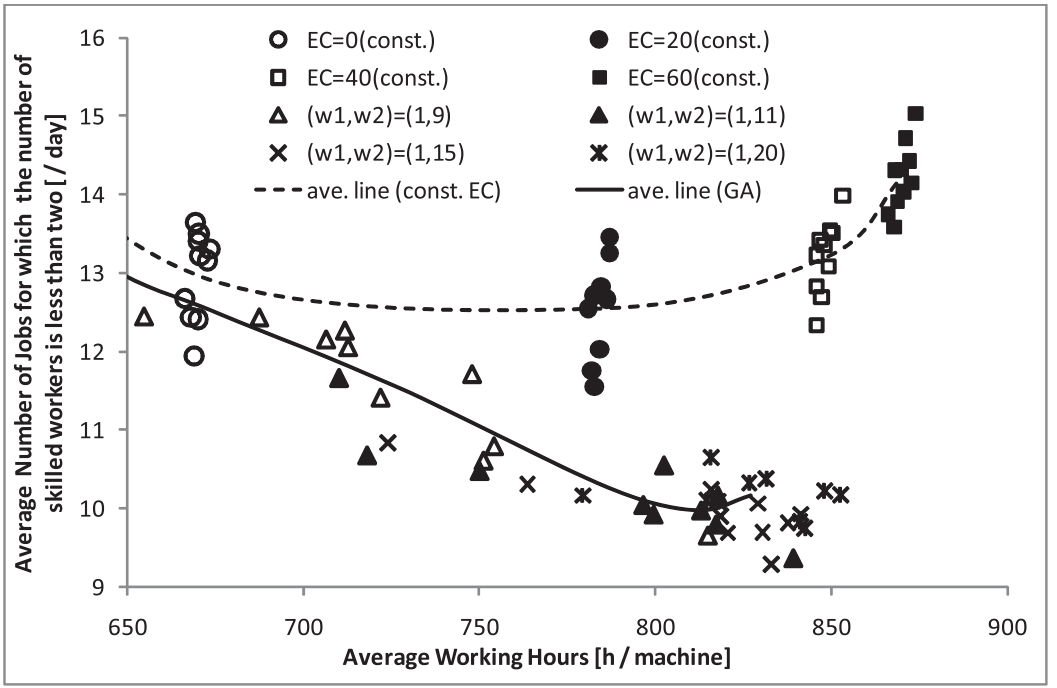

Fig. 6 Distribution of average solutions calculated using GA or using the constant EC values.

simulation 10 times. Each marker of the GA solutions (black marker) in the graph indicates the set of average solutions of the 10 individuals selected by performing GA 10 times. Each marker of the constant EC solutions (white marker) indicates the set of average solutions of ten results of the simulation using each constant EC. This graph shows that when the $\left(w_{1}, w_{2}\right)$ is equal to $(2,1)$ or $(1,1)$, the proposed method has obtained the solutions as good as the solutions calculated by using constant EC value of -40. The authors could not obtain solutions that had short average working hours than the solutions calculated by using constant EC value of -60 when we increased the value of $w_{1}$. In contrast, when $w_{2}$ is higher than $w_{1}$, especially the sets of $\left(w_{1}, w_{2}\right)$ are equal to $(1,11),(1,15)$, and $(1,20)$, the Pareto-optimal solutions obtained by using these weitht sets are better than the solutions of constant EC. Figure 6 shows the closeup of the section of $650-900$ [h / machine] of the average working hours. It also shows the distribution of average solutions $\left(\overline{x_{k}}, \overline{y_{k}}\right)$ calculated by using the sets of $\left(w_{1}, w_{2}\right)$ and GA or by using the constant EC values. Each marker indicates average solution $\left(\overline{x_{k}}, \overline{y_{k}}\right)$ of each selected individual. This graph shows the proposed method obtains many better solutions than the solutions obtained by using constant EC values.

Next, we observe the changes in the EC values of the good solutions obtained by the proposed method. Figure 7 shows the average EC values that the selected individuals store in their chromosomes. As described in the $\S 4.1$, the value of EC changes every time $N_{m} N_{j} / L_{c}=$ $100 \times 100 / 10=1000$ jobs are allocated. When the set of $\left(w_{1}, w_{2}\right)=(2,1)$, the values of EC are negative values and nearly flat. This is the reason why their solutions are as good as the solutions calculated by using constant EC value (-40). In contrast, when the set of $\left(w_{1}, w_{2}\right)=(1,15)$, the value of EC significantly changes. In this case, it sets the value of EC to a negative value at the beginning and the value gradually increases to increase the number of OJT. The value decreases again before the end of the period to decrease frequency of OJT. This is the reason why the individuals which have such EC values obtain much better solutions than the solutions calculated by using constant EC. It is impossible to execute such a strategy when the value of $\mathrm{EC}$ is constant.

\section{Discussion}

As described in $\S 5$, the proposed system calculated some solutions that were better than the solutions obtained by using a constant EC. This result shows the effectiveness of the proposed system which changes the value of EC depending on the number of finished jobs. However, the system calculates some solutions that are similar to the solutions computed by using 


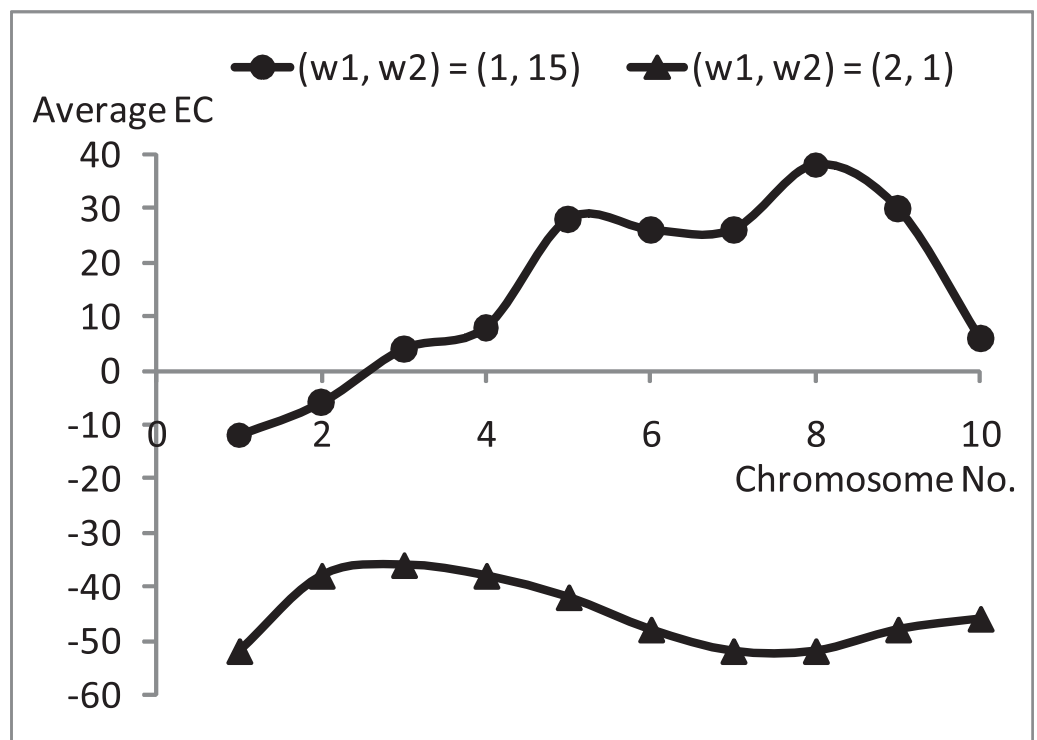

Fig. 7 Average EC values that the selected individuals store in their chromosomes

a constant EC (especially, the solutions that have short working hours). It would appear that it is necessary to construct a new system that changes the values of EC depending on the various situations (the current number of skilled workers, progress of maintenance, etc.) in order to obtain considerably better solutions. The final purpose of this research is to find effective rules for adjusting EC in order to realize a good balance between efficiency and education depending on the situation. In this paper, we have proposed an automatic EC tuning method using GA and have shown the usefulness of changing the value of EC during the long-term maintenance task as the first stage of this research. In the next stage, we aim to find effective rules for adjusting EC by analyzing the solutions calculated using GA.

\section{Conclusion}

In this paper, we proposed a simulation-based scheduling optimization system using a genetic algorithm. The purpose of this study was to adjust the value of EC automatically by considering the balance between working efficiency and skill education and to find effective rules for adjusting the value of EC. The experimental results showed that the proposed system could compute some solutions that were better than the solutions computed by using a constant EC.

\section{References}

( 1 ) Tateno, T. and Shimizu, K.,Work Scheduling by Use of Worker Model in Consideration of Learning by On-The-Job Training, Journal of Advanced Mechanical Design, Systems, and Manufacturing, Vol.1, No.3(2007), pp.430-441.

( 2 ) Jabor, M.Y. and Bonney, M., Production Breaks and the Learning Curve, The Forgetting Phenomenon, Applied Mathematical Modeling, Vol.20(1996), pp.162-169.

( 3 ) Goldberg, D.E., Genetic Algorithms in Search, Op-timization, and Machine Learning, Addison- Wesley(1989).

( 4 ) Smith, R.G., The Contact Net Protocol: High-Level Communication and Control in a Distributed Problem Solver, IEEE Transaction on Computers, Vol.C-29, No.12(1980), pp.1104-1113.

( 5 ) Tamaoki, H. Ochi, M. and Araki, M., A Genetics-Based Machine Learning Approach to Production Scheduling, Proceedings of IEEE International Symposium on Industrial Electronics, Vol.2(1998), pp.714-719. 